\section{Is a Single Provincial Pharmacy Program Beneficial for the Advancement of Pharmacy Practice?}

\section{THE "PRO" SIDE}

The provincial health authority in Alberta, Alberta Health Services (AHS), is the result of a merger in May 2008 of 9 regional health authorities, the Alberta Cancer Board, the Alberta Mental Health Board, and the Alberta Alcohol and Drug Abuse Commission. ${ }^{1}$ Since then, the previously existing regional pharmacy departments have become a single pharmacy program. The AHS pharmacy program has clearly benefited pharmacy practice in Alberta, despite challenges during the merger process. The creation of AHS was the largest health care merger in Canadian history, but lessons were taken from previous initiatives to create a provincial program, the most notable being cancer care. From the time when a provincial program was created for cancer care, in 1995, until the creation of AHS, the Alberta Cancer Board was responsible for all aspects of cancer services, including pharmacy, and demonstrated many improvements in pharmacy practice that were mirrored in the larger, more recent merger.

With this merger, pharmacy practice in the province of Alberta has advanced in numerous areas, 8 of which are described in more detail below.

\section{Provincial Perspective}

The single greatest benefit of a provincial pharmacy program is the broad perspective provided by pharmacy leaders from various areas of practice and geographic regions. Applying a provincial perspective to issues ensures that proposed initiatives can benefit the entire province. Economies of scale allow consideration of initiatives that would not be viable at the level of a regional authority or single site. Initiatives such as telepharmacy and robust drug distribution systems are examples of solutions more feasible at the provincial level than at the local scale. Pharmacist prescribing, previously endorsed by 2 of the health entities involved in the merger, will soon be recognized across AHS. A provincial perspective helps us move toward ensuring that all patients receive consistent pharmacy services throughout the province, irrespective of geographic place of residence.

\section{Budget}

The provincial pharmacy program now works directly with AHS, rather than with regional or site boards, to determine the program budget. This pan-provincial approach allows for consistent funding across the province, which in turn levels the playing field. Previously, if a regional authority did not consider the advancement of pharmacy practice to be a priority, pharmacy services were at risk of falling behind provincial standards.

\section{Provincial Formulary}

Having a provincial pharmacy program has facilitated the development and implementation of a single provincial drug formulary for acute care, as well as ongoing formulary management. The provincial Drug and Therapeutics Committee held its first meeting in March 2011. Although there are many benefits to this approach, transparency and consistency are the most apparent at these early stages.

Work on the provincial formulary was led by drug utilization evaluation pharmacists, who collected and collated formularyrelated information (e.g., formulary listings for acute care, therapeutic interchanges, restrictions, and guidelines) from the 9 former health regions and the Alberta Cancer Board for a total of 3322 drug products. ${ }^{2}$ The Drug and Therapeutics Committee now has provincial feedback and costing data for each medication that is being considered for inclusion in the formulary. Operationally, having one provincial formulary allows consistent access to medications across the province and improves inventory and contracting management, as well as operational workflow. Regardless of their location in the province, health care practitioners are aware of the therapeutic options available to their patients, and delays in treatment and pharmacy workload are decreased. Duplication of pharmacy workload related to the development of formulary policies and to assessment and maintenance of the formulary is reduced because the work is performed for the province as opposed to individual regions.

\section{Drug Information}

Before the AHS merger, drug information resources across the province varied widely among regional health authorities. With the provincial pharmacy program, all health care practitioners across AHS now have access to Lexi-Comp, Micromedex, and e-CPS. The overall cost of providing these resources to all front-line employees under the singular AHS umbrella is less than the cost had each of the former health authorities purchased their own licenses. Furthermore, the drug information centres in Calgary and Edmonton can now be accessed by all AHS employees. Prior questions and responses have been entered into a central repository, which can be quickly referenced in case similar questions are asked again, which eliminates unnecessary repetition of work. Eventually, all AHS employees will be able to search the database for previously answered questions, further decreasing the turnaround time on responses.

\section{Clinical Practice}

A provincial clinical program has fostered development of common expectations for individual pharmacists' practice and a common vision for clinical pharmacy practice. Each AHS pharmacist is matched with a Clinical Practice Leader, who acts as a resource to assist individual pharmacists to develop their respective professional advancement plans. Additionally, development of common 
training and orientation provides a level of consistency not achievable under the former regional authority model. Clinical presentations, previously booked primarily locally with the telehealth video-conferencing system, are now routinely available to all sites province-wide.

\section{Key Performance Indicators and Activity Measurement}

Using the same key performance indicators across all sites within the pharmacy program allows comparison of pharmacy services against established standards, as well as comparisons of sites with similar size, geographic limitations, or patient populations. These indicators assist in identifying gaps, as well as areas of strength, on the basis of objective criteria. Measurement of key performance indicators (in drug distribution, patient care, education and practice research, medication quality and safety, and customer service) can be used to assess and measure efficiencies realized through amalgamation of regional services.

\section{Stakeholder Engagement}

A single provincial program has allowed stakeholders external to AHS to connect with hospital pharmacy practice in Alberta. This common point of connection has improved outcomes of common initiatives and increased the interaction of the program with external stakeholders. For example, the streamlined relationship with the University of Alberta Faculty of Pharmacy and Pharmaceutical Sciences has facilitated training for all AHS preceptors. The faculty is now able to work with a single group of dedicated people throughout the province on this initiative, whereas it previously worked with at least 10 different groups to roll out preceptor training. The implementation of preceptor workshops has been more efficient because of the provincial model and one point of contact.

\section{Vision and Strategic Plan}

The ability to create a common vision and strategic plan is possibly one of the biggest benefits realized by having a single provincial program. Done correctly, this universal approach leads to a common destination for the entire program, and also ensures that growth of the best practices within the organization is not stalled while other practices work to catch up to the shared vision.

\section{Conclusion}

Overall, creating a provincial approach to health care delivery and a single pharmacy program has benefited pharmacy practice in Alberta. In our view, the overarching goal of AHS should be to grow the provincial pharmacy program and to create standards that enhance patient care and pharmacy practice, not to create a homogenous pharmacy program that settles on a middle ground. While the merger has been unquestionably challenging, the benefits to date have outweighed the disadvantages. As our provincial program moves forward, many more benefits are just starting to come to light.

Qualifying statement: In May 2011, AHS announced that its organizational structure would be realigned to enhance decision-making at the local and zonal level. The final structure for pharmacy is currently under discussion, but no major changes are anticipated. What AHS has made clear is that the gains associated with a provincial model, many of which have been outlined in the preceding article, must be maintained and must continue to be strengthened as we move forward.

\section{References}

1. Our history [website]. Edmonton (AB): Alberta Health Services; 2010 [cited 2011 April]. Available from: www.albertahealthservices.ca/191.asp

2. Alberta Health Services pharmacy therapeutics, drug utilization team. Alberta Health Services province-wide hospital formulary... Canadian first... made in Alberta! [poster]. Canadian Society of Hospital Pharmacists Banff Seminar; 2011 Mar 25-27; Banff (AB).

Jeremy Slobodan, BSP

Director, Practice \& Therapeutic Standards \& Support

Alberta Health Services

Red Deer, Alberta

Deb van Haaften, BScPharm, MA

Executive Director

Pharmacy Services, Edmonton and Area

Alberta Health Services

Edmonton, Alberta

Carole R Chambers, BSc(Pharm) MBA

Director, Cancer Services

Alberta Health Services

Calgary, Alberta

\section{THE "CON" SIDE}

For my part in this debate, I considered the question from an administrative perspective. And, as is typical for many administrative questions, the answer can be found in the phrase "It all depends."

To begin, we should ask why the profession should advance. The evolution of pharmacy practice has resulted from the visionaries who saw and still see the need for pharmacists, with all of their special knowledge and skills, to provide direct patient care within the safest and most cost-effective distribution system, without advancing simply for the sake of advancing. It is understood that no profession has value if it is totally self-serving. The question then becomes "Do the decision-makers and funders see advancement of the profession as a benefit to the patient or the public or merely as empire-building by the profession?"

To answer this question from an administrative perspective, we might consider how pharmacy practice has advanced in the past. History tells us that advancing practice starts with one person or perhaps a few individuals, usually working at a given site or in academia or both, who have a vision of where the profession should be, and it is this vision that leads to enhancement of the role of the pharmacist and the profession. The vision may be converted into action at a specific site and is often taken up by a voluntary professional association that then develops tools or guidelines to encourage and support practitioners in implementing change. Certainly, we have seen the profession advance over time, but it is a slow process that is limited by funding and a lack of will to advance from both internal and external perspectives.

Is there any evidence to demonstrate that centralization on a large scale actually enhances the profession? 
The stated reasons for regionalization, subsequent centralization, and back again are largely to reduce overhead, to rationalize services and thus avoid duplication, and to improve efficiency. But we must ask the question, "Has it been effective?" In reply, I must say that I see no correlation between these policy decisions and the advancement of any profession. It is understood that the goals of the health authorities and the payers are often in direct conflict with the aspirations of the health care professions. This is primarily because of the perception that the professions are always looking for more resources and, even though providing those resources may ultimately benefit the system, there are only so many to go around.

In the case of pharmacy, we have developed gold standards of practice through the work of the many volunteers and staff at the Canadian Society of Hospital Pharmacists. These standards represent goals for pharmacists and pharmacy managers to strive toward. However, in many jurisdictions, they are not legal standards. Therefore, it has always been a challenge to reach the goals, given funding levels within the public health system in Canada. Usually, health care organizations respond more quickly to legal standards than to gold standards. Even wellintended pharmacy managers struggle at times to implement needed change while trying to maintain current services. This is particularly true in times of government cutbacks.

Realistically, if the leaders in a provincial pharmacy department are visionary and want to advance practice, can they achieve this goal, given the mandate they have received from the government authority that oversees funding and overall policy decisions related to the provision of health care? It all comes down to the question, "Is bigger necessarily better?"

We all know that pharmacy services are complex and that leadership is required to support the front-line practitioners at every practice site. Yes, certain services can be centralized, particularly some of the drug distribution components, such as packaging and bulk production of IV admixtures. However, there will always be a need for front-line production. We also know that patient-focused care involves people looking after people. The care providers, while practising independently, also need support at the front-line level to be effective in their delivery of care. Thus, while a provincial department may attempt to standardize as much as possible, every facility will have its own particularities that will require customization to meet the needs of its patients.

Is a provincial pharmacy department able to respond quickly to the needs of the sites? Or does it have to seek approvals from a higher source of authority? Is this process any more efficient than the current system in many provinces? In a provincial system, are individual sites able to change practice through innovation and re-allocation of resources?

Recently, we have seen advances in practice as a result of the need to improve patient safety. These changes have come about as a result of pressure to reduce risk and avoid harm to the patient but also to avoid litigation against the corporation. Often, this has resulted in increased funding to support the safety agenda and to meet accreditation requirements; it has not been driven by a large central body that has undertaken to advance the profession.

I would argue that any profession advances in its own distinct ways, either through voluntary associations whose members volunteer their time to develop guidelines and tools for other members or, most likely, through innovators at individual practice sites who take up the challenge to improve patient care and who are willing to share their ideas with their peers.

Ultimately, the advancement of any profession depends on a vision, committed individuals, administrative support, and a belief that the advancement is necessary to improve the health of, and to reduce the risk to, the patient or the public. In today's environment of interprofessional collaboration, advancement of the profession will also depend on a multidisciplinary approach to enhance the patient care agenda.

This multidisciplinary approach can occur within a sitespecific department or a provincial department, as long as all of the pieces are in place and there is a commitment to move ahead. Notably, the commitment must come from all levels, including front-line staff, because even the best intentions on the part of a corporation may be met with resistance if the front-line staff do not buy in.

So the answer to the question of whether a single provincial pharmacy department is beneficial for the advancement of pharmacy practice is "It all depends on the vision, the will, and the perceived need to care for and protect the public, as well as on administrative support at whatever level." Advancement of the profession is not likely to be accomplished any better or any quicker by a provincial department than by a local institution. Advancement of the profession will depend on the vision and the will of those who want to make the change; it does not necessarily depend on the size of the organization.

Bill Wilson, BSP, FCSHP

Pharmacy Department

Mount Sinai Hospital

Toronto, Ontario

\section{Acknowledgement}

The author thanks Bill Bartle for reviewing this piece from the clinical perspective. 